\section{Fatores Que Podem Predizer a Estatura Final na Deficiência da 21-Hidroxilase}

$\mathrm{C}$ OM A INTRODUÇÃO DO GLICOCORTICÓIDE no tratamento da hiperplasia adrenal congênita por deficiência da 21-hidroxilase (HAC-21OH) houve melhora significativa na qualidade de vida e no prognóstico dos pacientes. O tratamento tem como um de seus objetivos a reposição de glicocorticóide a fim de normalizar os níveis de andrógenos e evitar o avanço da idade óssea, porém, sem afetar a velocidade de crescimento. Entretanto, este tênue balanço nem sempre é obtido, o que pode levar ao comprometimento da estatura final.

No período de crescimento o glicocorticóide de escolha é o de ação curta, pois suprime menos a liberação hipofisária do hormônio de crescimento. São rotineiramente usados o acetato de hidrocortisona (12 a 15 $\mathrm{mg} / \mathrm{m}^{2} /$ dia) e o acetato de cortisona $\left(18-20 \mathrm{mg} / \mathrm{m}^{2} /\right.$ dia); ambos devem ser divididos em três tomadas pela sua meia-vida curta. Apesar da reposição com glico e/ou mineralocorticóides, o resultado da altura final ainda deixa a desejar: na maior parte das casuísticas está entre -1 e -2 DP em relação à estatura alvo (1).

Numerosos esforços tem sido realizados na procura de fatores que possam ser determinantes do prognóstico da altura final, porém os resultados da literatura são controversos. A identificação destes fatores poderia contribuir para a precoce intervenção e na melhora do resultado da altura final. Lemos-Marini e cols. (2) seguindo esta linha, avaliaram em estudo retrospectivo os fatores que poderiam ter influenciado a altura final em 27 pacientes portadores da forma clássica ( $13 \mathrm{com}$ a forma perdedora de sal e $14 \mathrm{com}$ a forma virilizante simples). Os pacientes receberam hidrocortisona na dose de $18-26 \mathrm{mg} / \mathrm{m}^{2} /$ dia no primeiro ano de vida e após a fase de lactente, $13-21 \mathrm{mg} / \mathrm{m}^{2} /$ dia. Foram analisados fatores como sexo, severidade da forma clínica, idade de início do tratamento (antes e depois de 18 meses) e tempo de duração do tratamento $(<5$, entre 5 e 10 e $>10$ anos $)$, os quais não apresentaram diferença significativa no resultado da altura final. Entretanto, foi observada uma tendência dos pacientes do sexo masculino, com a forma virilizante simples e com tempo menor de tratamento apresentarem comprometimento maior da estatura final em relação a alvo. Em geral neste grupo de pacientes, a falta da crise de perda de sal ou a ausência de alterações da genitália ao nascimento, faz com que a idade ao diagnóstico seja tardia, época na qual já pode existir avanço importante da idade óssea. Neste último grupo a idade média no início do tratamento foi 7,2 anos e idade óssea 12,6 anos. Apesar também da falta de diferença com significado estatístico, foi indentificada tendência de prognóstico melhor de altura na forma perdedora de sal (com média de diagnóstico aos 30 dias) e nos casos com a forma viriliante em que a terapêutica foi instituída antes dos 18 meses de idade. Estes dados mostram que o diagnóstico precoce pode, provavelmente, intervir de forma positiva no prognóstico da altura. O aumento do número de casos poderá responder esta questão de forma definitiva.

\section{editorial}

Tânia A.S. Sanchez Bachega

Médica Assistente da Disciplina de Endocrinologia (Unidade de

Endocrinologia do Desenvolvimento e Laboratório de Hormônios e Genética Molecular LIM/42), Hospital das Clinicas da Faculdade de Medicina da

Universidade de São Paulo (USP), SP. Professora Colaboradora da Faculdade de Medicina da USP 
Em nosso serviço, durante o período de crescimento utilizamos o acetato de cortisona na dose de 18 - $20 \mathrm{mg} / \mathrm{m}^{2} /$ dia, dividido em 3 doses, com $20 \%$ da dose maior à noite, com o objetivo de otimizar a supressão noturna do ACTH. Na forma perdedora de sal utilizamos a 9 $\alpha$-fluor-hidrocortisona 150-200 $\mu \mathrm{g} /$ dia nos dois primeiros anos de vida e após, 50-100 $\mu \mathrm{g} /$ dia. Consideramos como bom controle hormonal a normalização dos níveis de androstenediona e de testosterona, dosados às 8:00 hs antes do recebimento da medicação. Em análise retrospectiva de 30 meninas portadoras da forma clássica que atingiram sua estatura final e que usaram o acetato de cortisona desde o diagnóstico, 15 destas apresentaram bom controle hormonal e 15 controle hormonal ruim por problemas de aderência. Entre os dois grupos não houve diferença significativa da altura final quanto à severidade da forma clínica. Provavelmente, isto decorre da presença da virilização da genitália externa no sexo feminino, que faz com que tenham seu diagnóstico e instituição da terapêutica precoce, como ocorre na forma perdedora de sal. Entretanto, observamos que o grau do controle hormonal foi um fator prognóstico da altura em nossa amostra. As meninas com controle bom apresentaram desvio padrão da altura final de $-1,0$ $\pm 0,9$ e as com controle ruim $-2,2 \pm 1,2(p<0,05)$. Todas as pacientes com bom controle atingiram o seu potencial genético de crescimento, a média do desvio padrão da altura alvo neste grupo foi $-0,8 \pm 0,8$.

Outro fator que poderia estar envolvido na altura final é o critério utilizado na determinação do controle hormonal. Vários autores preconizam a manutenção dos valores da 17-hidroxi-progesterona (17OHP) até $10 \mathrm{ng} / \mathrm{mL}$ para a forma clássica (1). É importante salientar que os níveis de 17OHP podem permanecem elevados na maioria dos casos nos quais os valores de andrógenos encontram-se em níveis prépúberes. Na nossa experiência, muitos casos que apresentam estes níveis da 17OHP possuem diminuição da velocidade de crescimento, o que indica supertratamento.

A literatura tem divulgado outros esquemas terapêuticos, os quais se encontram em fase de acompanhamento, com o objetivo de melhorar a aderência e/ou controle hormonal e preservar o potencial genético de crescimento. Caldato et al (2004) avaliaram os parâmetros auxológicos, hormonais e radiológicos em 23 pacientes com a forma clássica recebendo dose única matinal de fosfato de prednisonolona. Os autores utilizaram como controle 21 pacientes recebendo acetato de hidrocortisona $10 \quad-15 \mathrm{mg} /$ $\mathrm{m}^{2} /$ dia três vezes ao dia, com metade da dose maior pela manhã. Todos receberam adicionalmente fludrocortisona $100 \mu \mathrm{g} / \mathrm{dia}$. Os pacientes foram divididos na análise quanto ao sexo, idade e estadio puberal durante período de observação de um ano. O grupo que recebeu prednisolona apresentou menor avanço da idade óssea assim como melhora do desvio padrão da altura em relação à idade óssea em comparação ao grupo 2 , que recebeu hidrocortisona $(\mathrm{p}<0,02)$. $\mathrm{Na}$ avaliação ao final de um ano de seguimento pode ser observado que a prednisolona preservou a velocidade de crescimento. Se estes pacientes atingirem o seu potencial genético de altura, esta droga pode oferecer uma nova modalidade terapêutica, com regime posológico mais fácil e evitando a necessidade de manipulação de drogas, uma vez que a prednisolona é disponível comercialmente.

Recentemente, foi proposta a associação de hidrocortisona em doses baixas $\left(8 \mathrm{mg} / \mathrm{m}^{2} / \mathrm{d}\right)$, fludrocortisona com flutamida (inibidor da 5 a-redutase tipo 2 ) e testolactona (inibidor da aromatase) (4). Os resultados do acompanhamento de 28 crianças após dois anos de tratamento foram promissores, houve normalização da velocidade crescimento apesar dos elevados níveis de andrógenos e controle do avanço da idade óssea (D idade óssea $/ 2$ anos $=0,7 \pm 0,3$ anos). Os efeitos colaterais foram raros, porém é necessário aguardar os resultados da estatura final para a confirmação da eficácia deste tratamento.

Outro critério a ser analisado na HAC-21OH é o avanço da idade óssea. Pacientes que apresentam idade óssea de 12 anos podem apresentar puberdade precoce central, porém temos observado casos nos quais a puberdade iniciou com idade óssea de 9 anos. A falta de bloqueio concomitante da puberdade precoce também levará ao comprometimento da altura final. Estes pacientes são tratados com análogos do LHRH e geralmente observa-se desacelaração importante da velocidade de crescimento $(<3 \mathrm{~cm} / \mathrm{ano})$. Com o objetivo de preservar a altura final, tem-se proposto a utilização de hormônio de crescimento recombinante $(\mathrm{GHr})$, na dose de $0,15 \mathrm{UI} / \mathrm{kg} / \mathrm{dia}$, para os casos que apresentam predição de altura final menor do -1 DP em relação à altura alvo (5). Existe apenas um trabalho publicado na literatura, cujo DP da altura final foi $-0,4 \pm 0,8$ nos pacientes com este esquema terapêutico. Este resultado foi significamente melhor em relação ao grupo de pacientes que recebeu não recebeu $\mathrm{GHr}$.

Por fim, outra modalidade terapêutica da HAC$2 \mathrm{lOH}$ é a adrenalectomia bilateral (6), que foi principalmente indicada para meninas com a forma clássica, portadoras de mutações que abolem a atividade en- 
zimática e que apresentam controle hormonal difícil. A adrenalectomia bilateral tem como dados positivos, controle do hiperandrogenismo, redução da dose de hidrocortisona para duas vezes ao dia e diminuição do número de avaliações hormonais. Entretanto o autor deste trabalho, em comunicação pessoal, após alguns anos de seguimento notou aumento significante no número de crises adrenocorticais, o que leva ao questionamento desta modalidade terapêutica.

As novas formas de tratamento da HAC-21OH parecem promissoras, porém ainda estão em fase experimental e a evolução ao longo prazo é necessária para avaliar seu real benefício. Os trabalhos da literatura apresentam resultados controversos sobre quais fatores clínicos, laboratoriais e terapêuticos poderiam influir no prognóstico da altura na HAC-210H. Isto decorre do fato que as casuísticas apresentam diferentes tamanhos, formas de apresentação clínica, modalidades terapêuticas, assim como também diferentes padronizações para o critério de bom controle hormonal. Estes pontos devem levantar a discussão em nosso meio e estimular a criação de grupos de estudos multicêntricos com protocolos combinados, a fim de que possamos obter dados que nos permitam conclusões definitivas.

\section{REFERÊNCIAS}

1. White PC, Speiser PW. Congenital adrenal hyperplasia due to 21-hydroxylase deficiency. J Clin Endocrinol Metab 2000;21:245-91.

2. Lemos-Marini SHV, Guerra Jr G, Morcillo AM, Baptista MTM, Silva LO, Maciel-Guerra AT. Hiperplasia congênita das supra-renais por deficiência da 21-hidro- xilase: altura final de 27 pacientes com a forma clássica. Arq Bras Endocrinol Metab 2005;49/6:902-907.

3. Caldato MCF, Fernandes VT, Kater CE. One-year evaluation of single dose prednisolone therapy for 21-hydroxylase deficiency. Arq Bras Endocrinol Metab 2004;48:705-12.

4. Merke DP, Keil MF, Jones JV, Fields J, Hill S, Cutler Jr GB. Flutamide, testolactone, and reduced hydrocortisone dose maintain normal growth velocity and bone maturation despite elevated androgen levels in children with congenital adrenal hyperplasia. J Clin Endocrinol Metab 2000;85:1114-20.

5. Lin-Su K, Vogiatzi MG, Marshall I, Harbison MD, Macapagal MC, Betensky B, et al. Treatment with growth hormone and luteinizing hormone releasing hoemone analog improves final adult height in children with congenital adrenal hyperplasia. J Clin Endocrinol Metab 2005;90:3318 -25.

6. Van Wyk JJ, Gunther DF, Ritzen EM, Wedell A, Cutler Jr GB, Migeon CJ, et al. The use of adrenalectomy as a treatment for congenital adrenal hyperplasia. J Clin Endocrinol Metab 1996;81:3180-90.

Endereço para correspondência:

Tânia Sanchez Bachega Hospital das Clínicas, FMUSP,

Disciplina de Endocrinologia Caixa Postal 3671.

01060-970 - São Paulo, Brasil

Fax: 5-11-3069-7519

e-mail: tbachega@usp.br 